\title{
Novel technical modifications of hand-assisted laparoscopic dissection of a large obturator mass: A case report and literature review
}

\author{
JUN ZHANG $^{1}$, PENG ZHONG ${ }^{2}$, HAIPENG TONG ${ }^{3}$, JUN JIANG ${ }^{1}$ and DALI TONG ${ }^{1}$ \\ Departments of ${ }^{1}$ Urology, ${ }^{2}$ Pathology and ${ }^{3}$ Radiology, Daping Hospital, \\ Army Medical University, Chongqing 400042, P.R. China
}

Received January 23, 2020; Accepted June 5, 2020

DOI: 10.3892/ol.2020.12132

\begin{abstract}
Laparoscopic lateral pelvic tumor dissection (LLPTD) for transobturator tumors may be technically challenging due to the requirement for sufficient operative space. The present study discusses a technique modification with combination of the laparoscopic approach and hand-assisted (HA) open surgery for patients with large obturator masses. LLPTD was performed using the combined approach, defined as HA-LLPTD, with one case treated. According to this technique, a combined working space is constructed based on the outreached laparoscopic space and open extraperitoneal approach, followed by HA-LLPTD. Finally, a literature review was performed to retrospectively evaluate 17 cases of obturator tumors, in terms of tumor type and operative approach. The tumor in the present case was successfully and completely resected, without any obvious intra- and post-operative complications. Based on the literature review, the majority of the cases were benign $(\sim 75 \%)$ and originated from neurological tissue $(\sim 50 \%)$. The selection of the operative approach was either open or minimally invasive (50\% each). HA-LLPTD allows experienced urological laparoscopic surgeons to safely and completely perform obturator surgery without obstruction of the obturator foramen or formation of intraperitoneal adhesions and associated complications. Therefore, HA-LLPTD may be more useful for transobturator tumor resection compared with the conventional intraperitoneal approach.
\end{abstract}

\section{Introduction}

Obturator masses have a certain probability of occurrence in pelvic tumors. The most common origins of obturator masses

Correspondence to: Dr Dali Tong or Professor Jun Jiang, Department of Urology, Daping Hospital, Army Medical University, 10 Changjiangzhilu, Chongqing 400042, P.R. China

E-mail: tongdali1985@163.com

E-mail: jiangjun_64@163.com

Key words: laparoscopic approach, hand-assisted laparoscopic dissection, transobturator tumor are neurological tumors, including schwannoma and neurofibroma (1-3). Local tumors around the lateral obturator zone have been reported to be potentially resectable by laparoscopic lateral pelvic tumor dissection (LLPTD) (4). However, only a limited number of studies have assessed and confirmed the safety and operability of such tumors (4). Careful inspection of $\mathrm{CT}$ and MRI is important to determine the tumor positioning preoperatively. The tumors, locating in the retroperitoneal space, may be close to iliac vessels, obturator nerve and even spermatic cords $(1,2,5)$. In order to gain better exposure for tumor dissection, exploratory transperitoneal laparoscopy has usually been performed to treat affected cases (4). Resection of the tumor was carefully performed to ensure the safe removal of the specimen with complete and functional preservation of the iliac vessels and obturator nerve $(2,6,7)$. In particular, cross-obturator masses may be technically demanding due to the requirement for sufficient operative space. The aim of the present study was to introduce a technique modification with the combination of the laparoscopic approach and hand-assisted (HA) open surgery for patients with large obturator masses.

The present study reported on the use of a novel operation procedure for coping with trans-obturator tumor, combining laparoscopic with open approaches. Due to the limitation of obturator obstruction and operation space, laparoscopic surgery alone is not able to fully dissociate obturator tissue to reach the tumor area below the obturator. In order to ensure the overall removal of the tumor, the most appropriate method used in the present study is to push the tumor to the area above the obturator for laparoscopic dissection. This procedure requires open surgical assistance. Compared with traditional open surgery, the present method utilizes minimally invasive laparoscopic surgery and fine dissection with fewer complications for fast recovery, and due to these advantages, it may be is deemed more appropriate than the usual clinical approach to meet the patient's clinical requirement.

\section{Case report}

Case details. In January 2018, a 53-year-old male patient was admitted to the Department of Urology, Daping Hospital, Army Medical University (Chongqing, China) with a 1-month 
history of abdominal discomfort. The patient was subsequently referred to the Department of Urological Oncology for resection of the mass. Ultrasound examination revealed a right pelvic space-occupying lesion. No obvious palpable lymph nodes were detected. The laboratory examination and chest X-rays were normal. A contrast-enhanced CT scan (Fig. 1) revealed a heterogeneously enhanced mass, measuring $35 \times 37 \times 76 \mathrm{~mm}$, located in the right pelvic space, with infiltrative growth, extending into and passing through the obturator foramen, $4 \mathrm{~cm}$ above and $3 \mathrm{~cm}$ below the obturator fossa, neighboring the pelvic wall. Lymph node involvement was not detected on CT examination. The suspected preoperative diagnosis included vagal nerve schwannoma, fibroid tumor, paraganglioma, inflammatory pseudotumor or non-Hodgkin lymphoma. The baseline characteristics of the patient are presented in Table I. The clinical samples and images were provided by the Department of Pathology and Medical Imaging following approval by the Research Ethics Committee of Daping Hospital, Army Medical University (Chongqing, China) and obtaining written informed consent from the patient. The treatment plan was discussed by the multidisciplinary board of the hospital.

Surgical technique. The surgical procedure was based on the combination of laparoscopic and open surgery. The mass was planned to be removed by transabdominal laparoscopic dissection with the aim of achieving better exposure, complete dissection and sufficient hemostasis to stem bleeding.

First, a small $15-\mathrm{mm}$ incision was made above the umbilicus. The anterior rectus sheath was then incised, followed by parting the rectus abdominis muscle exactly in the middle. A $12-\mathrm{mm}$ trocar was inserted into the abdominal space through the posterior rectus sheath, which was used as the camera port. Two $12-\mathrm{mm}$ ports were also inserted laterally to the rectus abdominis muscle, one on each side.

The procedure was performed according to the previous description for LLPTD surgery (4). The external iliac vein was first exposed upon identifying the pubic bone and Cooper's ligament as anatomical landmarks, which was followed by opening the peritoneum and separating the vessels in the obturator canal. The tumor completely occupied the obturator space and the obturator nerve was closely associated with the tumor. The obturator nerve was carefully isolated and preserved. The internal iliac vessel branches supplying the tumor, including artery and vein, were dissected and then clamped.

However, during surgery, the upper part of the tumor extending above the obturator fossa fully occupied the obturator space; therefore, there was insufficient space for exploring and excising the lower part of the tumor below the obturator foramen. In order to solve this problem and achieve complete resection, an open exploratory incision was made on the inner side of the inguinal ligaments in order to push the lower part of the tumor upwards. By combining the laparoscopic and open routes, the tumor was fully excised. No intraoperative complications occurred (Fig. 2). The dissected mass was placed into the sample bag and extracted through the $12-\mathrm{mm}$ port trocar.

Outcome. The perioperative baseline information of the patient is presented in Table II. The operative time was $210 \mathrm{~min}$. The estimated blood loss was $200 \mathrm{ml}$. R0 resection was achieved.
Table I. Characteristics of the patient.

\begin{tabular}{lc}
\hline Item & Value/description \\
\hline Sex & Male \\
Age $($ years $)$ & 53 \\
BMI $\left(\mathrm{kg} / \mathrm{m}^{2}\right)$ & 30.5 \\
Operation & Laparoscopic LPTD and Open \\
Histology & Solitary fibrous tumor \\
Pathological stage & I \\
\hline
\end{tabular}

BMI, body mass index.

Table II. Perioperative data.

Item

Value/description

Operative time ( $\mathrm{min})$

210

Blood loss (ml)

Adjacent structures removed en bloc

Post-operative complications

200

None

Low intestinal

obstruction/weakness

of the adductor

muscle

Post-operative hospital stay (days)

10

Laparoscopic imaging revealed an $8-\mathrm{cm}$ mass spanning the obturator fossa and compressing the obturator nerve. The postoperative hospital stay was 11 days. There were no severe postoperative complications except transient low intestinal obstruction and weakness of the adductor muscle.

The cut surface of the tumor had a white to tan appearance. Hematoxylin and eosin staining and immunohistochemical examination revealed significantly increased cellular proliferation, locally scattered mast cells, 2 mitotic figures per 10 high-power fields and positive CD117, CD34 and STAT6 staining, findings consistent with solitary fibrous tumor (SFT), which is a mesenchymal neoplasm without typical symptoms (Fig. 3). The patient reported postoperative short-term dysfunction of hip joint adduction and right leg pain due to mild obturator nerve damage during the dissection. At the hospital, the patient received timely neurotrophic therapy based on methylcobalamin. The 2-month follow-up revealed no residual disease based on $\mathrm{CT}$ evaluation.

\section{Literature review}

A total of 186 articles were retrieved by searching the PubMed database for studies published between January 13, 2006 and December 20, 2019 using the search term 'mass in obturator'. Publications were included in the review if they met the following list of preset inclusion criteria: i) The mass was located in the obturator fossa; ii) the mass was a solid tumor based on surgical biopsy diagnosis or imaging diagnosis; iii) lymph node or tumor metastasis was excluded. A total of 17 

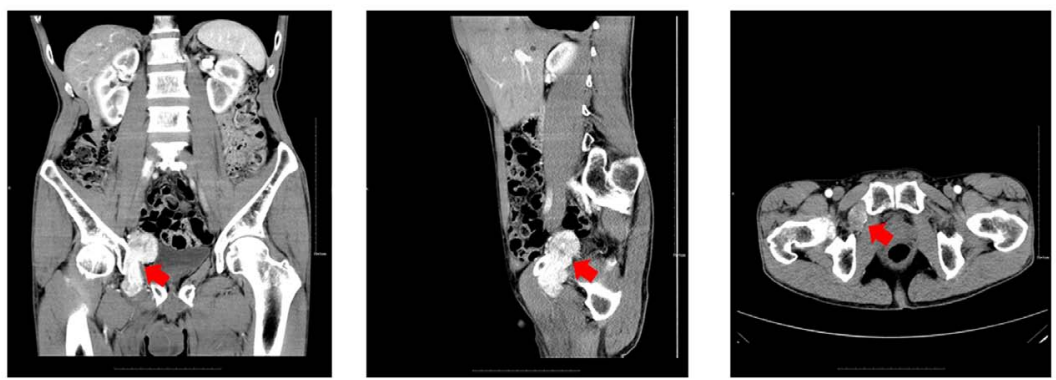

Figure 1. Representative abdominal CT findings. Red arrows indicate tumor location, which spans the obturator region, respectively indicated on coronal plane, sagittal plane and transversal plane from left to right.
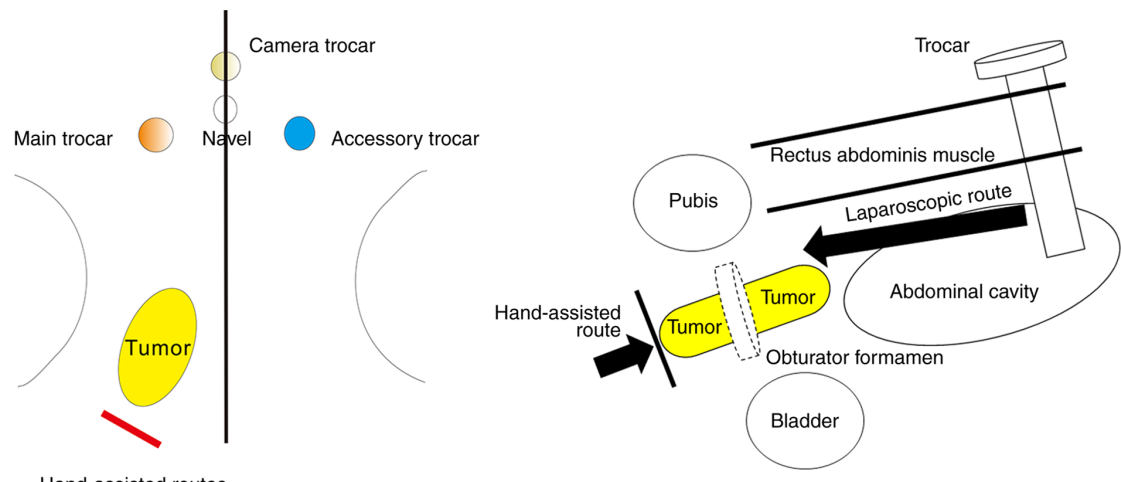

Hand-assisted routes

Figure 2. Schematic illustration of port placement and combined operative approaches. Left panel: Port plan with location of camera trocar, operation trocar and open incision. Right panel: Anomorphic surface of combined operative approaches.

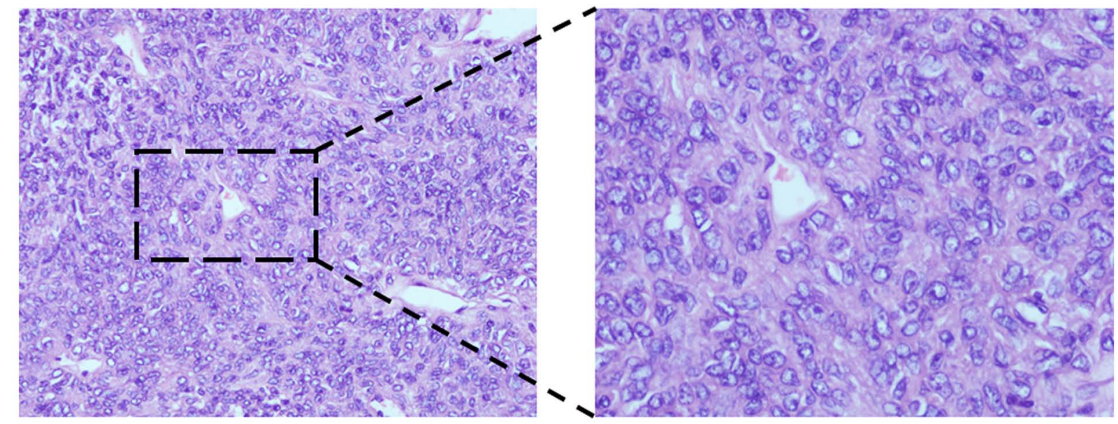

Figure 3. Hematoxylin and eosin staining of the tumor specimen displayed with low and (x200) high-power (x400) magnification, respectively. The pathological diagnosis is solitary fibrous tumor (SFT).

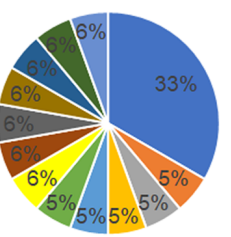

- Obturator nerve schwannoma

- Symptomatic parasitic leiomyoma

- Neurofibroma

- Presacral lipoma

- Aggressive angiomyxoma

- Pelvic angiomatosis

Thigh abscess

- Mucoid pseudocyst of the obturator nerve

- Nonseminomatous germ cell tumor

- Solitary fibrous tumor

- Malignant schwannoma of the obturator

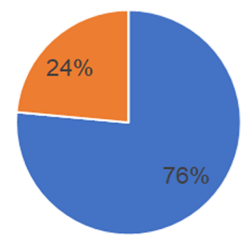

-Benign

" Malignant

(n)

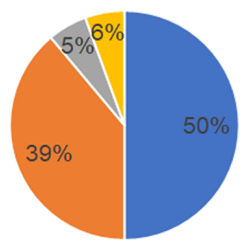

- Neurological

" Genitourinary

- Mesenchymal

- Other

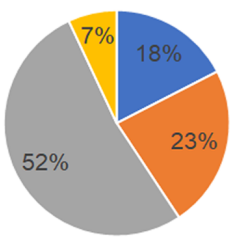

- Robotic

- Laparoscopic

- Open

" Laparoscopic+Open

Figure 4. Constituent ratio analysis of different types of tumors, tumor characteristics, tissue origin and operative method, based on literature review reported in the previous literature and included in Table III. 


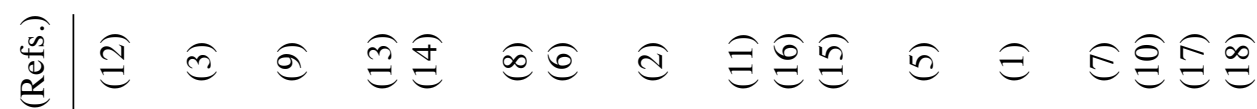

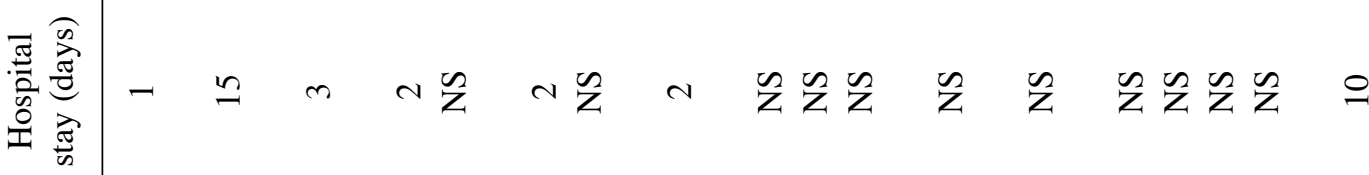

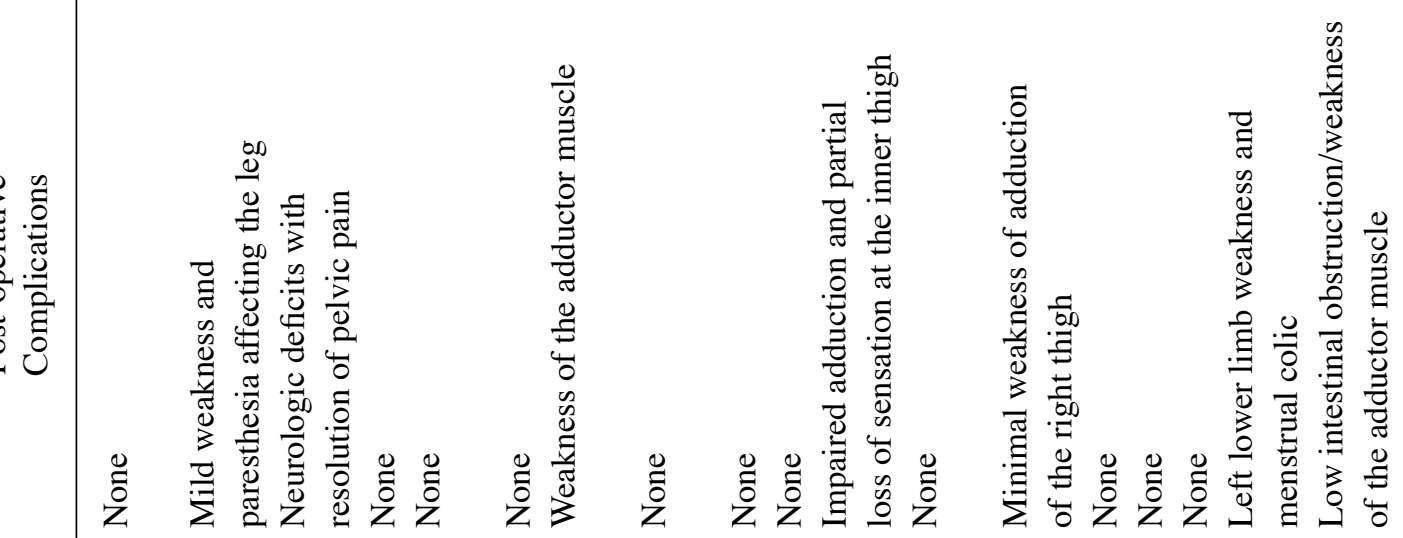

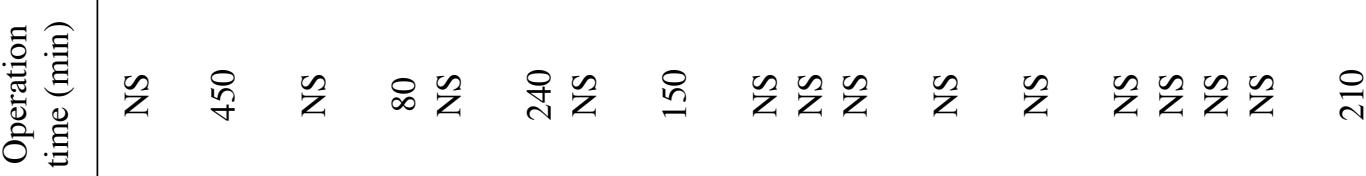

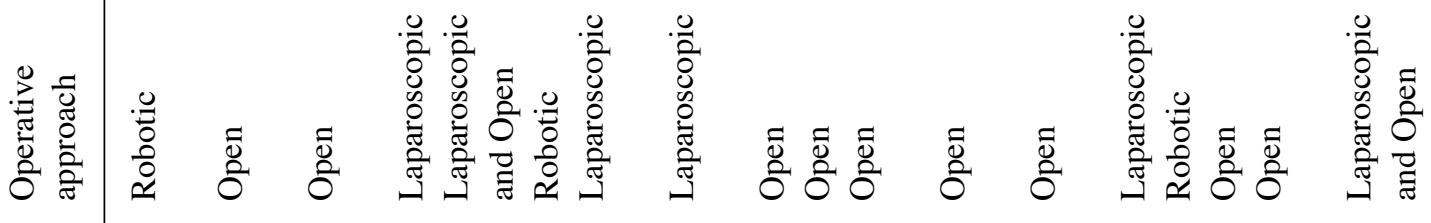

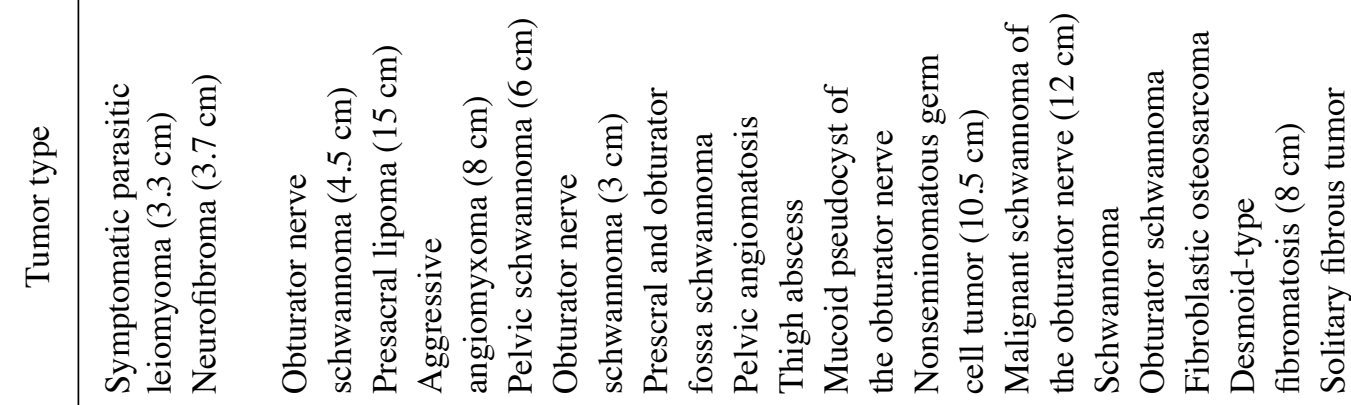

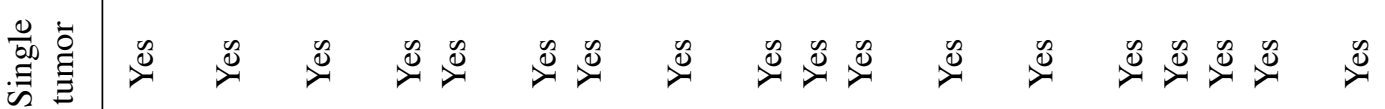
离

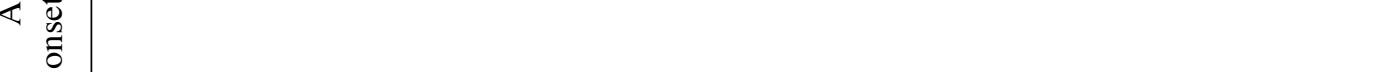

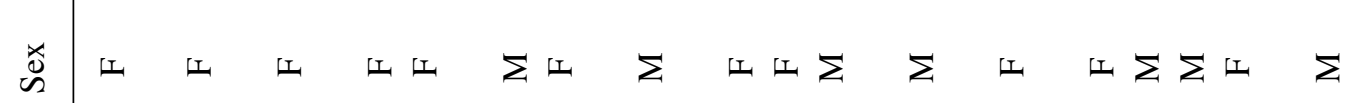

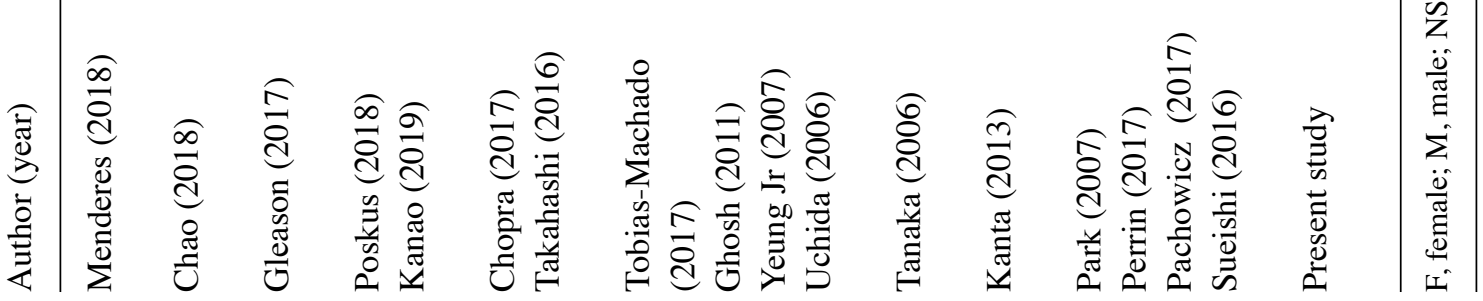


publications, mainly case reports, were included in the review of the present study. A total of 6 cases were diagnosed as obturator nerve schwannoma $(2,6-10)$ and 1 case was a malignant schwannoma of the obturator nerve treated with neoadjuvant chemotherapy followed by neurosurgery (1). Other types of tumor (1 case each) included retroperitoneal extragonadal germ cell tumor (5), pelvic angiomatosis (11), parasitic leiomyoma (12), neurofibroma (3), presacral lipoma (13), aggressive angiomyxoma (14), mucoid pseudocyst of the obturator nerve (15), thigh abscess mistakenly diagnosed as sarcoma (16), fibroblastic osteosarcoma (17) and desmoid tumor (18). The patients' characteristics are summarized in Table III. From the previous studies and the present study, data on the cases of obturator tumor were extracted and further analyzed based on composition ratio and are presented in Table IV and Fig. 4. The majority of the cases were benign $(\sim 75 \%)$ and originated from neural tissue $(\sim 50 \%)$. The selected operative approach was either open or minimally invasive (50\% each).

\section{Discussion}

Laparoscopic surgery provides surgeons with a clear and magnified view of the operative field (19). Therefore, it is useful for complex surgical procedures, particularly in deep locations in the narrow lateral pelvic space $(4,14)$. It appears that only a limited number of studies have explored surgical dissection of obturator masses and these lacked systematic conclusions $(2,5)$.

LLPTD, as a minimally invasive pelvic surgical technique, has been applied in the clinical setting due its feasibility and reduced associated blood loss (4). However, the effect of the limited space must be taken into consideration. In the present case, occupation of the obturator fossa by a large tumor made LLPTD more difficult. The combined open approach with HA-LLPTD allows experienced LLPTD surgeons to modify the tumor location and perform complete tumor dissection without any complications (14).

This approach may be performed via surgical cooperation and without the requirement for an assistant port (1). LLPTD combined with an open approach has been previously reported $(14,20)$. Surgeons must have a comprehensive anatomical understanding to use this approach. The technique reported in the present study included LLPTD combined with the open method for pelvic and inguinal surgery, and it appears to be more useful for such cases as compared with the conventional approach.

Obturator nerve injury is the most common surgical complication reported in the literature $(6,15)$. The reasons are as follows: i) The combination of sharp and blunt dissection of the tumor causes injury to the nerve, as it is situated in close proximity to the tumor; ii) tumor tissue may be dissected along with the obturator nerve, irrespective of whether the nerve is infiltrated by the tumor; iii) pelvic lymph node dissection may cause injury. It is noteworthy that caution should be taken intraoperatively to protect the nerve against mechanical and thermal injury; in addition, the treatment process should include close observation of perioperative symptoms and signs, postoperative follow-up and neurotrophic support $(1,3)$.

Recent studies have reported on cases of SFT located in the pelvic region $(21,22)$. However, to the best of our knowledge,
Table IV. Different types of tumors reported in the previous studies included in Table III.

Tumor type $\mathrm{N}$

Obturator nerve schwannoma

6

Symptomatic parasitic leiomyoma

Neurofibroma

Presacral lipoma

Aggressive angiomyxoma

Pelvic angiomatosis

Thigh abscess

Mucoid pseudocyst of the obturator nerve

Non-seminomatous germ cell tumor

Solitary fibrous tumor

Malignant schwannoma of the obturator

Desmoid-type fibromatosis

Fibroblastic osteosarcoma

\section{1}

1

1

1

1

1

1

1

1

1

SFTs spanning the obturator fossa with involvement of obturator nerve have not been reported to date. SFTs may be misdiagnosed as other tumor types occurring in this area, such as neurological tumors. Furthermore, the assessment of malignancy should incorporate the biological behavior of the tumor and not rely on histopathological examination alone. Atypical SFTs may undergo malignant transformation and recurrence (23). Therefore, a rigorous follow-up is crucial for assessing the efficacy of surgical treatment.

Several types of tumor have been reported to be located in the obturator fossa, the majority of which are benign, mainly derived from neurological and mesenchymal tissues $(7,12)$. In particular, obturator nerve schwannoma is more commonly reported compared with other types. The operative approach may be either open or minimally invasive $(1,6,7,9)$. However, in recent years, robotic and laparoscopic surgery have become mainstay surgical options $(6,8)$. In the present case, a combined approach was used to treat a transobturator tumor, which appeared to be the optimal choice.

HA-LLPTD allows experienced laparoscopic surgeons to safely perform complete resection of tumors located in the obturator fossa with fewer complications. The combination of laparoscopic and open surgery is an innovative method for the excision of obturator foramen masses, which provides clinical solutions for extracting tumors of the obturator region. Although the short-term outcome appears to be favorable, due to the small sample size, further investigations with larger sample sizes are still required to assess and explore the long-term outcome of this approach.

\section{Acknowledgements}

Not applicable.

\section{Funding}

This work was supported in part by the Dali Tong Chongqing Science and Technology Bureau (Basic and Frontier Research 
Project; grant no. cstc2018jcyjA2133) and the Chongqing Municipal Health and Health Committee (Science and Health Joint Medical Research Project; grant no. 2018QNXM041).

\section{Availability of data and materials}

The datasets used and/or analyzed during the present study are available from the corresponding author upon reasonable request.

\section{Authors' contributions}

DT acquired the data, performed the literature review, performed the surgery and pre-operative administration, and drafted, reviewed and edited the manuscript for intellectual content. JJ contributed to the conceptualization, analysis of the present study and revised the manuscript for important intellectual content. JZ performed the surgery, contributed to data and image analysis and manuscript editing. PZ made pathological staining and recognize pathological diagnosis. HT took radiological images and recognize radiological diagnosis. All authors have read and approved the manuscript.

\section{Ethics approval and consent to participate}

The present study was approved by the Research Ethics Committee of Daping Hospital, Army Medical University. The patient provided informed consent.

\section{Patient consent for publication}

Written informed consent was provided by the patients for the publication of the study, including personal data and images in all formats.

\section{Competing interests}

The authors declare that they have no competing interests.

\section{References}

1. Kanta M, Petera J, Ehler E, Prochazka E, Lastovicka D, Habalova J, Valis M and Rehak S: Malignant schwannoma of the obturator nerve. Bratisl Lek Listy 114: 584-586, 2013.

2. Tobias-Machado M,Hidaka AK, Sato LLK, Silva IN, Mattos PAL and Pompeo ACL: Laparoscopic resection of prescral and obturator fossa schwannoma. Int Braz J Urol 43: 566, 2017.

3. Chao WT, Liu CH, Chen YJ, Wu HH, Chuang CM and Wang PH: Neurofibroma involving obturator nerve mimicking an adnexal mass: A rare case report and PRISMA-driven systematic review. J Ovarian Res 11: 14, 2018.

4. Masubuchi S, Okuda J, Hamamoto H, Ishii M, Osumi W, Yamamoto M, Inoue Y, Tanaka K and Uchiyama K: Totally extraperitoneal approach to laparoscopic lateral lymph node dissection for patients with recurrent lateral pelvic lymph nodes after rectal cancer surgery: A novel technique-M TEP LLND. Surg Today 49: 981-984, 2019.
5. Tanaka T, Kitamura H, Masumori N, Tsukamoto $\mathrm{T}$ and Kimura M: Retroperitoneal extragonadal germ cell tumor presenting as a bulky pelvic mass of the obturator fossa. Int $\mathbf{J}$ Urol 13: 180-182, 2006.

6. Takahashi H, Hara M, Tsuboi K, Sagawa H, Ishiguro H, Matsuo Y and Takeyama H: Laparoscopically resected obturator nerve schwannoma: A case report. Asian J Endosc Surg 9: 307-310, 2016.

7. Park NY, Chong GO and Lee YS: Laparoscopic resection of schwannoma in the anomaly of obturator nerve. J Laparoendosc Adv Surg Tech A 17: 769-773, 2007.

8. Chopra S, Dharmaraja A, Satkunasivam R and Gill IS: Robot-assisted laparoscopic resection of a pelvic schwannoma. Urol Case Rep 11: 63-65, 2017.

9. Gleason T, Le BH, Parthasarathy K and Robinson-Bennett B: Obturator nerve schwannoma as a mimic of ovarian malignancy. Case Rep Obstet Gynecol 2017: 9724827, 2017.

10. Perrin H, Brunner P, Ortega JC, Mercier B, Clement N, Robino C and Chazal M: Robotic resection of an obturator schwannoma with preservation of normal nerve fascicles and function. J Robot Surg 11: 479-483, 2017.

11. Ghosh SB, Mala YM, Tripathi R and Singh A: Pelvic angiomatosis: An unusual cause of recurrent obstructed labor: A case report and review of literature. Arch Gynecol Obstet 283: 127-129, 2011.

12. Menderes G, Nhundu B, Levy K and Silasi DA: Robotic resection of a symptomatic parasitic leiomyoma from the obturator fossa. J Minim Invasive Gynecol 25: 23, 2018.

13. Poskus E, Makunaite G, Kubiliute I and Danys D: Case report: Laparoscopic approach in the treatment of presacral lipoma. Ann Med Surg (Lond) 35: 64-66, 2018.

14. Kanao H, Aoki Y, Tanigawa T, Matoda M, Okamoto S, Nomura H, Omatsu K, Kato K, Utsugi K and Takeshima N: En bloc resection of an aggressive angiomyxoma by a novel combination laparoscopic and open perineal approach. J Minim Invasive Gynecol 26: 598-599, 2019.

15. Uchida A, Horiguchi A, Ide H, Hatakeyama N, Yoshimura I and Ogawa Y: Mucoid pseudocyst of the obturator nerve. Int J Urol 13: 471-472, 2006.

16. Yeung P Jr, Sokol A, Walton B and Iglesia C: Thigh abscess mistaken for sarcoma following transobturator tape: A case report and literature review. J Minim Invasive Gynecol 14: 657-659, 2007.

17. Pachowicz M, Drelich-Zbroja A, Szumilo J, Skwarcz S and Chrapko B: A mysterious tumor in the obturator internus muscle-a case report. Nucl Med Rev Cent East Eur 20: 62-63, 2017.

18. Sueishi T, Arizono T, Nishida K, Hamada T and Inokuchi A: A case of spontaneous regression of recurrent desmoid tumor originating from the internal obturator muscle after delivery. World $\mathrm{J}$ Oncol 7: 75-80, 2016.

19. Goel M, Kurunkar SR, Kanetkar A and Patkar S: Outcome of robot-assisted radical cholecystectomy in a high-volume tertiary cancer center in India. J Laparoendosc Adv Surg Tech Part B Videoscop 29: vor.2018.0539, 2019.

20. Blank JJ, Gibson EK, Peterson CY, Ridolfi TJ and Ludwig KA: Retroileal anastomosis in hand-assisted laparoscopic left colectomy: Experience at a single institution. Surg Endosc 34: 3408-3413, 2020.

21. Wang Y, Wei R, Ji T, Chen Z and Guo W: Surgical treatment of primary solitary fibrous tumors involving the pelvic ring. PLoS One 13: e0207581, 2018.

22. Fernandez A, Conrad M, Gill RM, Choi WT, Kumar V and Behr S: Solitary fibrous tumor in the abdomen and pelvis: A case series with radiological findings and treatment recommendations. Clin Imaging 48: 48-54, 2018.

23. Huang SC and Huang HY: Solitary fibrous tumor: An evolving and unifying entity with unsettled issues. Histol Histopathol 34: 313-334, 2019. 